\title{
Kommentar zur Stellungnahme des VEMS zum Bericht des Swiss Medical Board
}

Urs Metzgera,

Hans Bohnenblust ${ }^{b}$, im Namen des Fachgremiums Swiss Medical Board

a Professor em., ehem. Chefarzt Chirurgie, Triemlispital

b Dipl. Ing. ETH, M.S. MIT, (Technology Assessment)
In seiner Stellungnahme wirft der Verein Ethik und Medizin Schweiz (VEMS) dem Swiss Medical Board eine unseriöse Arbeitsweise vor. Der VEMS geht dabei von einem falschen Verständnis des Auftrags für den Fachbericht «Computertomographie-Scanner in der Abklärung der koronaren Herzerkrankung» vom 31. Dezember 2012 aus, obwohl alle Prämissen im Bericht ausdrücklich deklariert sind. Die Analysen des Swiss Medical Board sind immer auf präzise formulierte Fragestellungen ausgerichtet. Im vorliegenden Fall werden nicht - wie offensichtlich vom VEMS angenommen - die allgemeinmedizinischen und kardiologischen Diagnose- und Behandlungswege bei Verdacht auf eine koronare Herzerkrankung analysiert. Der Bericht beschränkt sich auf den Vergleich der kardialen Computertomographie mit den neuesten Geräten (NGCCT New Generation Cardiac Computer Tomography) und der invasiven Koronarangiographie bei bestimmten Patientengruppen, wenn im
Fachgesellschaften vorgeschlagene Fachspezialisten konsultiert. Diese äusserten sich sowohl zu den medizinischen Fakten wie auch zu den Kostenannahmen. Die im Fachbericht dargestellten Kosten der kardialen Computertomographie entsprechen wie explizit dargestellt - denjenigen, die das UniversitätsSpital Zürich typischerweise mit den Krankenkassen verrechnet. Zum Zeitpunkt der Berichterstattung waren die «Baserates» im SwissDRG-System noch unsicher; in einigen Kantonen waren sie lediglich mittels superprovisorischer Verfügung geregelt. Von daher wäre es nicht zielführend gewesen, einen Durchschnitt der Kosten über alle Kantone zu errechnen. Auf den exemplarischen Charakter der vorgelegten Kostenberechnungen wird im Fachbericht ausdrücklich hingewiesen. Die künftige Verfügbarkeit verbindlicherer Kostendaten würde zu einer Überprüfung der Kostenberechnungen und allfälliger Auswirkungen auf die Empfehlungen führen.

\section{Der Bericht des SMB beschränkt sich auf den Vergleich der beiden Verfahren bei bestimmten Patientengruppen, wenn im Rahmen des Diagnoseprozesses zwischen beiden Alternativen zu wählen ist.}

Rahmen des Diagnoseprozesses zwischen diesen beiden Alternativen zu wählen ist. Dies entspricht der exakten Fragestellung der Untersuchung des britischen NICE (National Institute for Health and Clinical Excellence), die der Arbeit des Swiss Medical Board in diesem Falle zugrunde liegt. Ein zentrales Anliegen der Trägerschaft war es nämlich, im Rahmen dieses Berichts zu prüfen, inwiefern sich ausländische HTA-Berichte zu einer gegebenen Fragestellung auf die schweizerischen Verhältnisse übertragen lassen. Das Swiss Medical Board hat denn auch bei diesem Fachbericht auf eigene Literaturrecherchen zur medizinischen Evidenz verzichtet und lediglich in einigen Punkten (zum Beispiel zu den Nebenwirkungen und der Strahlenbelastung) ergänzende Überlegungen angestellt. Die Empfehlungen im Bericht beziehen sich ausschliesslich auf den zuvor genannten Vergleich.

Die Vorwürfe betreffend eine mangelnde Zusammenarbeit mit den Fachgesellschaften und Fehler bei der Kostenermittlung sind nicht gerechtfertigt. Wie bei jedem anderen Fachbericht hat das Swiss Medical Board von der FMH bzw. den betroffenen
Die in der Stellungnahme des VEMS zitierten Passagen zur Kassenpflicht der Leistungen stammen aus dem Kapitel 6 des Fachberichts und dienen der Erläuterung der rechtlichen Rahmenbedingungen innerhalb der Obligatorischen Krankenpflegeversicherung (OKP). Sie sind nicht Gegenstand der Empfehlungen.

Letztlich möchten wir darauf hinweisen, dass der Vergleich zwischen der kardialen Computertomographie und der invasiven Koronarangiographie in der Diagnostik notgedrungen die Interessen der betroffenen Berufsgruppen berührt, d.h. der Radiologen auf der einen und der Kardiologen (vertreten im VEMS) auf der anderen Seite.

\section{Interaktiver Artikel}

Wollen Sie diesen Artikel kommentieren? Nutzen Sie dafür die Kommentarfunktion in der OnlineVersion oder sehen Sie nach, was Ihre Kolleginnen und Kollegen bereits geschrieben haben: www.saez.ch/aktuelle-ausgabe/interaktive-beitraege/ 